Research Article

\title{
Research on the Distribution Rule of Percussion Response Pulse Width in Coin-Tap Test
}

\author{
Jian Li $\mathbb{D}^{1,2}$ Liping Xu $\left(\mathbb{D},{ }^{1,2}\right.$ Xiaowen Yu, ${ }^{1}$ Jianjun Peng, ${ }^{1,2}$ and Lei Huang ${ }^{1}$ \\ ${ }^{1}$ School of Mechatronics Engineering, Henan University of Science and Technology, Luoyang 471003, China \\ ${ }^{2}$ Collaborative Innovation Center of Machinery Equipment Advanced Manufacturing of Henan Province, \\ Luoyang 471003, China
}

Correspondence should be addressed to Liping Xu; xlpzz@163.com

Received 15 February 2021; Accepted 20 August 2021; Published 29 August 2021

Academic Editor: Chengwei Fei

Copyright (C) 2021 Jian Li et al. This is an open access article distributed under the Creative Commons Attribution License, which permits unrestricted use, distribution, and reproduction in any medium, provided the original work is properly cited.

\begin{abstract}
The coin-tap method performs nondestructive testing by measuring the width of the tapping response pulse on the surface of the material. Existing studies have shown that defects in the material will cause the width of the tapping response pulse to increase. However, experiments have confirmed that different detection positions in the defective area will show different values of the width of the tapping response pulse, and the physical laws behind it have not been studied yet. To discuss its physical meaning, a mathematical model of the defective area is established, a method for calculating the width of the tapping response pulse is proposed, and a composite honeycomb structure with preset defects is used for data testing. Both the test results and the calculation results show that the pulse width of the tapping response will decrease with the increase of the defect depth and the deviation of the tapping position from the defect center. The consistency between the calculated results and the experimental results shows that the established defect model and pulse width calculation method can better explain the distribution law of the pulse width of the tapping response in the defective area.
\end{abstract}

\section{Introduction}

Composites have become the most widely used materials in industries such as aviation and aerospace due to their excellent specific strength, specific stiffness, and fatigue resistance. However, the composite material may have defects or damages during curing, forming, and using, which will cause the composite material structure and function to fail and even cause major accidents [1]. Therefore, nondestructive testing of composite materials is very important.

Coin-tap testing is one of the main methods of nondestructive testing in the aerospace industry. It has been automated in recent years and has become the first line of defense for composite material testing [2-4]. The researcher uses the acceleration sensor to collect the tapping response signal and analyses the time history of the impact force to determine the defect. The higher the value of the pulse width of the impact force at the tap point, the more serious the defect at that point $[5,6]$. As a measure of the degree of defect, the width of the tapping response pulse is essentially a description of the stiffness of the inspection point. Research shows that the main factors affecting the stiffness of the detection point are the diameter and depth of the damaged area. The shallower depth and larger diameter of the damaged area will reduce the stiffness of the damaged area [7]. However, the physical meaning behind it is not clear. There is still no precise definition of the mathematical relationship between various influencing factors and the pulse width of the tapping response.

To study the pulse width distribution of tapping response in the defective area, it is necessary to calculate the impact deflection at any point in the defective area. Abrate analysed the impact dynamics mathematical model between the object and the composite material and proposed the selection method of the spring-mass model under different impact conditions [8]. According to the thin plate bending theory, Feng and Yu studied the calculation method of the stiffness of the laminated plate low-speed impact model 
based on the spring-mass model [9]. Li et al. used the point source function method to solve the bending problem of circular plates under noncentrosymmetric loads $[10,11]$. Dong et al. used the Poisson formula of the circular plate and the natural boundary integral equation to obtain the bending solution of the circular plate under an asymmetric load [12]. Although researchers have conducted a lot of research studies from different angles, they have not been able to fully explain the distribution rule of pulse width. On the basis of existing research, this paper establishes a new physical model for the defective area and predicts the pulse width of the tap response. In the experiment, tapping tests were carried out for defective areas of different diameters and different depths. The test results are in good agreement with the predicted calculation results. It indicates that the new physical model and calculation method can better explain the pulse width distribution law of the tapping response.

\section{Theoretical Model of Coin-Tap Test}

The low-speed impact response of composite materials is a quasi-static response. Cawley pointed out that the existence of defects leads to changes in the stiffness of the material, which causes changes in impact characteristics. Therefore, when tapping the defective area and the sound area, different tapping sounds will be produced [5]. Based on the springmass model, he proposed a physical model for coin-tap test [13]. It is shown in Figure 1.

During the inspection process, due to the certain pressure between the hammer head and the inspected structure, there is a contact stiffness between the hammer head and the inspected structure. The impact force is transmitted to the tested structure through the spring with stiffness coefficient $k_{c}$. Cawley models the defects inside the material as a spring with stiffness $k_{d}$, and the value of $k_{d}$ in the sound area is close to infinity. The stiffness model of the defective area can be regarded as a series connection of two springs with stiffness coefficients $k_{d}$ and $k_{c}$. And, the effective stiffness of the defective area can be calculated with the following equation:

$$
k=\frac{k_{c} k_{d}}{k_{c}+k_{d}} .
$$

When an object with mass $m$ hits a spring with stiffness $k$ at velocity $v$, the motion control equation of this model is

$$
m \ddot{x}+k x=0 .
$$

The general solution is

$$
x(t)=C_{1} \cos \sqrt{\frac{k}{m}} t+C_{2} \sin \sqrt{\frac{k}{m}} t
$$

or expressed as

$$
x=A \sin \left(\sqrt{\frac{k}{m}} t+\alpha\right) .
$$

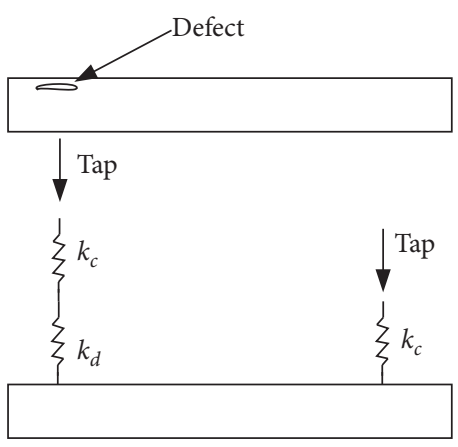

FIgURE 1: The physical model for the coin-tap test.

In the formula, $C_{1}, C_{2}, A$, and $\alpha$ are arbitrary constants, determined by the initial conditions. The relationship between them is determined by the following equations:

$$
\begin{aligned}
& A=\sqrt{C_{1}+C_{2}}, \\
& \alpha=\arctan \frac{C_{1}}{C_{2}} .
\end{aligned}
$$

The general solution of the vibration equation shown in equation (4) is the same as the simple harmonic vibration [14]. The amplitude of system vibration is $A$, and the phase at the time $t=0$ is $\alpha$. It can be seen that the angular frequency of system vibration is

$$
\omega=\sqrt{\frac{k}{m}}
$$

Therefore, the duration of the impact force generated during the impact is

$$
t=\frac{T}{2}=\frac{\pi}{\omega}=\pi \sqrt{\frac{m}{k}} .
$$

In the formula, $T$ represents a complete vibration period. It can be seen from equation (7) that the tapping response pulse width measured during the detection process is actually a description of the effective stiffness of the detection point. By solving the effective stiffness $k$ of each point in the defective area under the impact load, the pulse width distribution of the percussion response in the defective area can be obtained.

\section{Calculation of Effective Stiffness of Defective Area}

Most of the interlayer interfaces of composites are fragile, so defects or damages are prone to occur during manufacturing and use [15]. The defects of the laminate structure often appear in layers, and the skin and the core of the honeycomb sandwich structure may be debonded. These damages will reduce the rigidity of the structure, which can be effectively detected by the coin-tap test method. The common defect forms of different composite material structures are shown in Figure 2. 


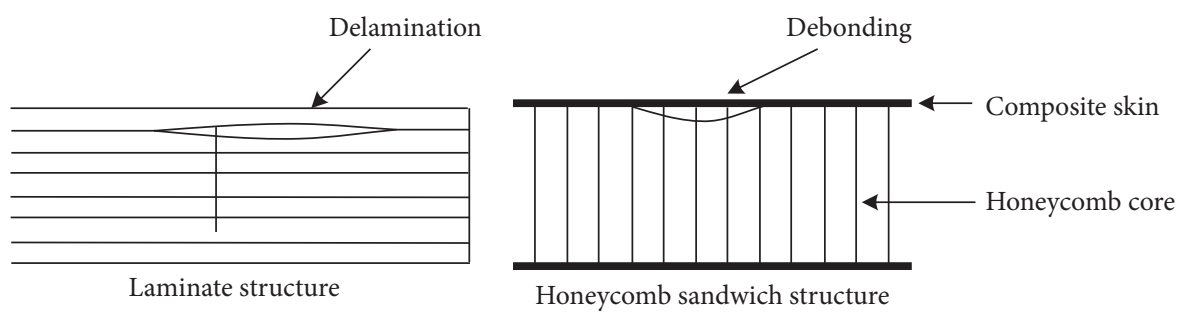

Figure 2: The common defect forms of different composite material structures.

Existing studies have shown that the physical phenomenon behind the coin-tap test can be explained by the principle of membrane resonance [16-19]. When the composite laminate is delaminated or the skin of the honeycomb sandwich structure is debonded from the core, the material layer above the defective area can be imagined as a thin plate bound by its edge [20]. Assuming that the radius of the defective area is $R$, when the lateral concentrated force $F$ acts on the defective area, the force analysis of the section is shown in Figure 3.

According to Kirchhoff hypothesis, the middle surface of the plate does not produce strain during the bending deformation process and always remains a neutral curved surface. Each point in the midplane has only a displacement $w$ perpendicular to the direction of the midplane, and on any normal line of the midplane, all points in the full thickness of the sheet have the same displacement $w$ [21, 22]. Therefore, the impact of shear deformation is ignored during the tapping process, and only the effect of bending deformation is considered. The effective stiffness of the round sheet at the striking point can be obtained by dividing the force $F$ at that point by the displacement $w$ at that point.

A circular thin plate with a radius of $R$ is provided, and its plane projection is shown in Figure 4.

Point $A$ and point $B$ are any two points on the circular thin plate. Assuming that the distance from center $O$ to point $A$ is $\overline{O A}=a$ and the distance from center $O$ to point $B$ is $\overline{\mathrm{O} B}=b$, then point $A$ and $B$ can be expressed as $A\left(a, \theta_{0}\right)$ and $B(b, \theta)$ in the polar coordinate form.

Since the edge of the circular plate is fixed, the deflection $w$ of the edge of the plate is 0 , and the slope $(\partial w / \partial x)$ of the elastic curved surface is 0 . It can be seen that the boundary conditions are

$$
\begin{aligned}
(w)_{b=R} & =0 \\
\left(\frac{\partial w}{\partial x}\right)_{b=R} & =0
\end{aligned}
$$

When a lateral concentrated force $F$ acts on point $A$, according to the calculation method in [12], the deflection formula of point $B$ can be obtained as

$$
w_{B}=\frac{F}{D} G\left(b, \theta ; a, \theta_{0}\right)
$$

In the formula, $G\left(b, \theta ; a, \theta_{0}\right)$ is the Green function of the reharmonization equation inside the unit circle, and its form is

$$
\begin{aligned}
G= & \left\{\frac{a^{2}+b^{2}-2 a b \cos \left(\theta-\theta_{0}\right)}{16 \pi R^{2}}\right. \\
& \left.\times \ln \frac{a^{2}+b^{2}-2 a b \cos \left(\theta-\theta_{0}\right)}{R+a^{2} b^{2}-2 a b \cos \left(\theta-\theta_{0}\right)}+\left(R-a^{2}\right)\left(R-b^{2}\right)\right\} .
\end{aligned}
$$

$D$ is the bending stiffness of the circular thin plate, and its calculation formula is

$$
D=\frac{E h^{3}}{12\left(1-v^{2}\right)} \text {. }
$$

In the formula, $E$ is the elastic modulus of the defective upper plate, $h$ is the depth of the defect, and $v$ is Poisson's ratio of the defective upper plate.

When point $A$ and point $B$ coincide, that is, $a=b$ and $\theta=0$, we can obtain

$$
w_{B}=w_{A}=\frac{F\left(R^{2}-b^{2}\right)^{2}}{16 \pi D R^{2}} .
$$

The effective stiffness of the impact point can be obtained as

$$
k=\frac{F}{w}=\frac{16 \pi D R^{2}}{\left(R^{2}-b^{2}\right)^{2}} .
$$

The formula expounds the specific mathematical relationship between the effective stiffness of the impact point and the depth and radius of the defective area, which is a new finding.

\section{Experiments and Discussion}

To verify the model and algorithm, a honeycomb sandwich structure with internal predefects was selected for the cointap test. The physical figure of the honeycomb sandwich structure is shown in Figure 5.

The length of the specimen is $400 \mathrm{~mm}$, the width of the specimen is $300 \mathrm{~mm}$, the height of the specimen is $30 \mathrm{~mm}$, and the thickness of the carbon fiber is $1 \mathrm{~mm}$. Its Poisson's ratio $v$ is 0.31 , and its elastic modulus $E$ is $210 \mathrm{GPa}$.

There are three built-in debonding defects at the position marked in Figure 5, and the parameters are shown in Table 1.

In the test, 5 inspection points were selected in defect 1 to measure the tapping response pulse width, while 9 inspection points in defect 2 and defect 3 were selected to 


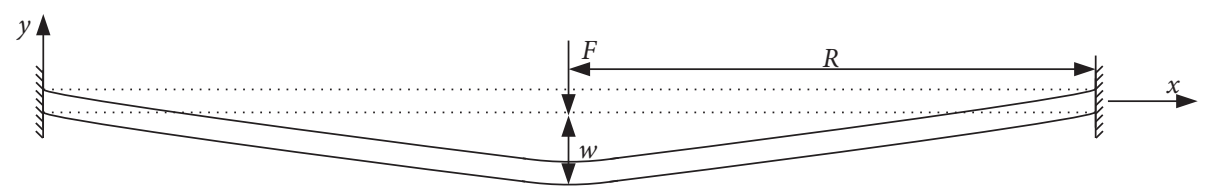

FIgURE 3: The force analysis of the section.

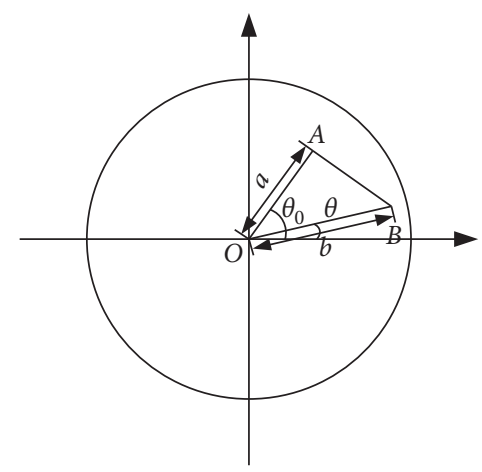

FIgURE 4: The plane projection of a circular thin plate.

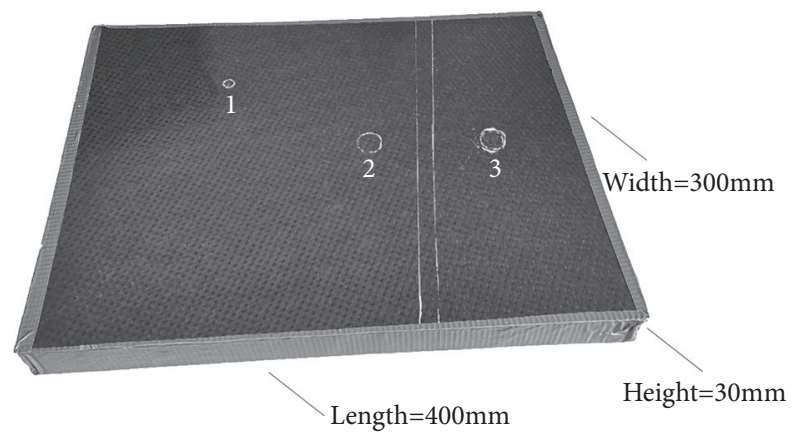

Figure 5: The honeycomb Sandwich structure with built-in defects.

TABle 1: Parameters of three defects.

\begin{tabular}{lcc}
\hline & Diameter $(\mathrm{mm})$ & Depth $(\mathrm{mm})$ \\
\hline Defect 1 & 10 & 0.5 \\
Defect 2 & 20 & 0.3 \\
Defect 3 & 20 & 0.5 \\
\hline
\end{tabular}

measure the tapping response pulse width. As shown in Figure 6, the nine white points marked are the locations of the nine tapping detections. These inspection points are evenly distributed along the diameter of the defective area, and each adjacent white point is spaced $2 \mathrm{~mm}$ apart.

In the test, an energized solenoid is used to drive a hammer with a mass of $33 \mathrm{~g}$ to strike, and the upper end of the hammer is connected to a spring. The tapping device used in the test is shown in Figure 7.

The hammer head is made of steel and has a curvature radius of about $2 \mathrm{~mm}$. When the solenoid is energized, the hammer head will accelerate the impact on the surface of the material. When the solenoid is de-energized, the spring drives the hammer head back to the initial position. When

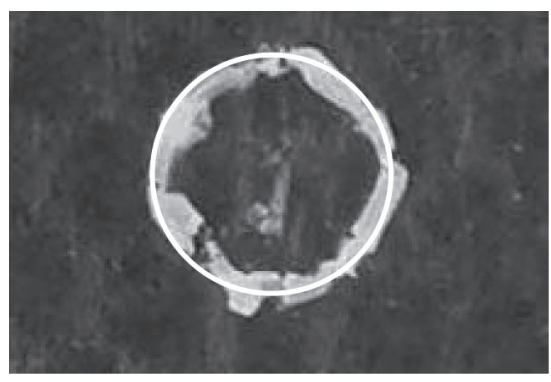

Figure 6: Schematic diagram of detection point location.

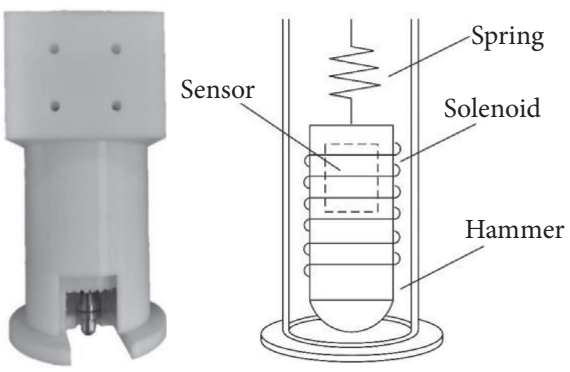

FIgURE 7: The tapping device and its schematic diagram.

the hammer collides with the structure, the speed of the hammer is about $0.2 \mathrm{~m} / \mathrm{s}$, and its impact force is about $36 \mathrm{~N}$. The tapping response signal is recorded by the piezoelectric acceleration sensor and transmitted to complete the pulse width measurement. The tapping signal is collected through piezoelectric acceleration sensors and data acquisition cards. The type of acceleration sensor is CA-YD-155. The signal of the data acquisition card is NI-USB-6009. Its analog input resolution is 14 bits, and the signal acquisition frequency is set to $48 \mathrm{KHz}$.

In the test, the detection positions are arranged in order along the direction indicated by the arrow in Figure 6, where point 5 is the center point of the defective area. The pulse width values of different points obtained by the experiment are shown in Table 2.

In Table 2, $|b|$ represents the distance between the detection position and the center of the defective area. The test results show that when the size of the defective area is larger, the tapping response pulse value is larger. In the defective area of the same size, when the distance between the defective position and the center of the defective area is the same, the larger the depth of the defect, the lower the width of the tapping response pulse. When the defect depth is constant, the tapping response pulse width at the center of the defective area is the widest. As the detection position moves away from the center of the defective area, the tapping 
TABLE 2: Pulse width measurement results at different points in the defective area.

\begin{tabular}{lccccccccc}
\hline & \multicolumn{7}{c}{ Pulse width (ms) } \\
\hline Serial number & 1 & 2 & 3 & 4 & 5 & 6 & 7 & 8 & 9 \\
$|b|(\mathrm{mm})$ & 8 & 6 & 4 & 2 & 0 & 2 & 4 & 6 & 8 \\
Defect 1 & & & 0.167 & 0.188 & 0.230 & 0.188 & 0.167 & \\
Defect 2 & 0.563 & 0.688 & 0.813 & 0.875 & 0.938 & 0.896 & 0.813 & 0.646 \\
Defect 3 & 0.271 & 0.313 & 0.375 & 0.438 & 0.459 & 0.438 & 0.396 & 0.292 & 0.542 \\
\hline
\end{tabular}

TABLE 3: Measured values of tapping response pulse width at different positions in the defective area.

\begin{tabular}{lccccccccc}
\hline & \multicolumn{7}{c}{$k\left(1 \times 10^{6} \mathrm{~N} \cdot \mathrm{m}^{-1}\right)$} \\
\hline Serial number & 1 & 2 & 3 & 4 & 5 & 6 & 7 & 8 \\
$|b|(\mathrm{mm})$ & 8 & 6 & 4 & 2 & 0 & 2 & 4 & 6 & 8 \\
Defect 1 & & & 37.526 & 6.89 & 4.86 & 6.89 & 37.526 & \\
Defect 2 & 2.026 & 0.641 & 0.372 & 0.285 & 0.263 & 0.285 & 0.372 & 0.641 \\
Defect 3 & 9.381 & 2.968 & 1.723 & 1.319 & 1.216 & 1.319 & 1.723 & 2.968 & 9.381 \\
\hline
\end{tabular}

TABLE 4: Calculated values of tapping response pulse width at different positions in the defective area.

\begin{tabular}{lccccccccc}
\hline & \multicolumn{7}{c}{ Pulse width (ms) } \\
\hline Serial number & 1 & 2 & 3 & 4 & 5 & 6 & 7 & 8 & 9 \\
$b(\mathrm{~mm})$ & 8 & 6 & 4 & 2 & 0 & 2 & 4 & 6 & 8 \\
Defect 1 & & & 0.080 & 0.189 & 0.225 & 0.189 & 0.080 & \\
Defect 2 & 0.349 & 0.620 & 0.814 & 0.930 & 0.969 & 0.930 & 0.814 & 0.620 \\
Defect 3 & 0.162 & 0.288 & 0.378 & 0.432 & 0.450 & 0.432 & 0.378 & 0.288 & 0.162 \\
\hline
\end{tabular}

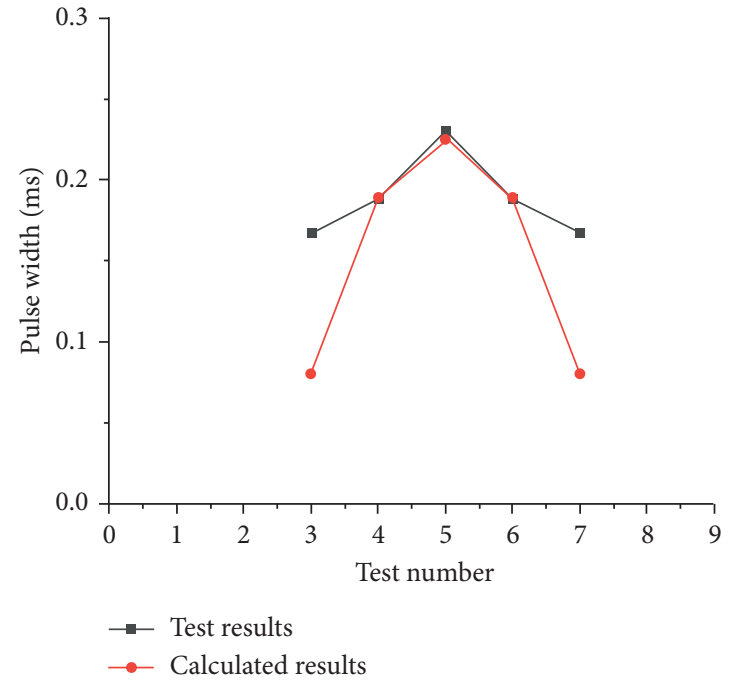

(a)

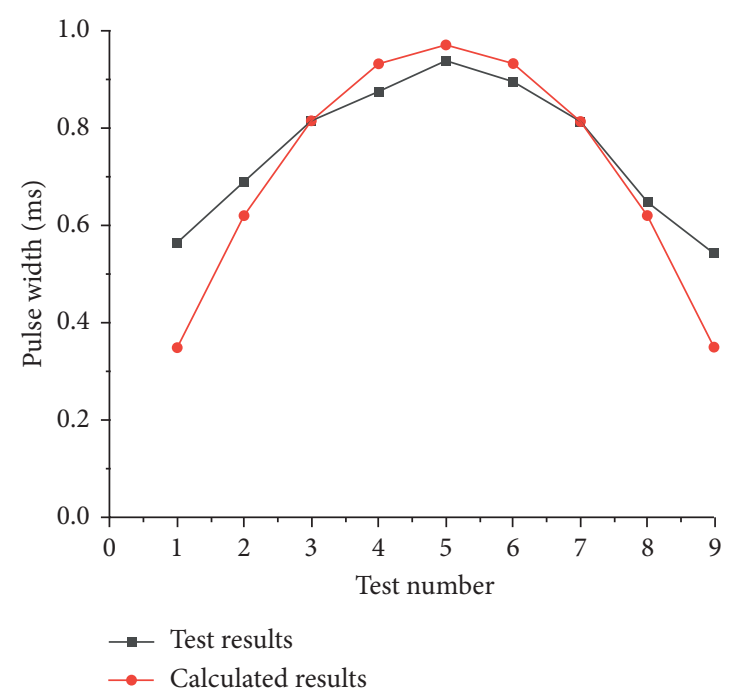

(b)

Figure 8: Continued. 


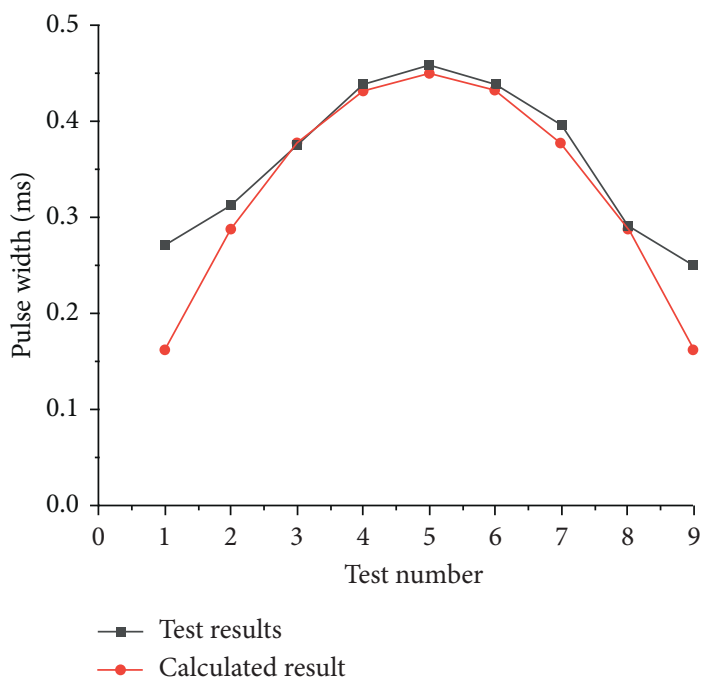

(c)

FIGURE 8: Curve comparison chart of test results and calculated results. (a) Defect 1. (b) Defect 2. (c) Defect 3.

response pulse width gradually decreases. It should be noted that when the distance between the detection position and the center of the defective area is the same, the measured values of pulse width are very close but not the same. The reason for the slight difference in pulse width at these positions may be the stiffness error on the surface of the test piece. Even if the detection is performed in a sound area, the tapping response pulse width will not all be the same. The values of the pulse width are usually concentrated in a certain range; such an error will not cause too much influence on the detection result.

According to equation (11), the bending stiffness of the plate can be obtained as

$$
D=\frac{E h^{3}}{12\left(1-v^{2}\right)}=0.523 \mathrm{~N} \cdot \mathrm{m}^{-1} \text {. }
$$

We can calculate the stiffness $k$ of different positions in the defective area, as shown in Table 3.

According to equation (7), the tapping response pulse width of the detection points at different distances from the center of the defective area can be calculated, as shown in Table 4.

The comparison result of the pulse width distribution curve obtained by the experiment and the calculated pulse width distribution curve is shown in Figure 8.

It can be seen from the calculation results that when the depth of the defect increases, the tapping response pulse width decreases significantly. This is because when the defective area is far away from the material surface, the thickness of the material layer above the defective area increases, resulting in an increase in the bending stiffness of the layer. The increase of the defect depth will greatly reduce the sensitivity of the coin-tap test method. When the defect depth is constant, the tapping response pulse width at the center of the defective area is the highest. As the detection position moves away from the center of the defective area, the tapping response pulse width gradually decreases. It can be seen from Figure 8 that the calculated pulse width distribution curve has a very similar changing trend to the experimental pulse distribution curve.

The data shows that the calculated results and the test results show good agreement near the center of the defective area. However, when the detection position is close to the edge of the defect, there is a big difference between the calculated result and the test result. This is because the material layer above the defective area in the test is not an absolute fixed support constraint, and the edge of the defect will show a tiny corner during the coin-tap test. When the detection position is very close to the edge of the defect, this slight change will cause the calculation error to be enlarged. However, the calculation result will not be too much affected in the main area.

\section{Conclusions}

In this paper, a mathematical model of the defective area is established for the coin-tap test method, and a method for calculating the tapping response pulse width of the defective area is proposed. The specific mathematical relationship between the effective stiffness of the tapping point and the depth and diameter of the defective area is described. A percussion test was carried out using a composite honeycomb structure with preset defects of different diameters and different depths, and the test results were compared and analysed with the calculated results. Experiments and calculations have shown that when the depth of the defect increases, the width of the tapping response pulse is greatly reduced; when the depth of the defect is constant, the width of the tapping response pulse in the center of the defective area is the highest. As the detection position moves away from the center of the defective area, the width of the tapping response pulse gradually decreases. The test results are in good agreement with the calculated results, indicating that 
the mathematical model and calculation method for the stiffness of the defective area proposed in the article better illustrate the distribution law of the pulse width of the tapping response in the defective area. This provides theoretical guidance for the application and research of the coin-tap test method and is of great significance to its further development. The disadvantage is that the tiny corner of the defect edge leads to a certain error in the calculation result of the position of the defect edge attachment. This will be further discussed in the future research.

\section{Data Availability}

The data supporting the findings of the study are included in the relevant figures and tables within the article.

\section{Conflicts of Interest}

The authors declare that they have no conflicts of interest regarding the publication of this paper.

\section{Acknowledgments}

This research was supported by the National Key Research and Development Project of China (no. 2018YFB1701200).

\section{References}

[1] S. P. Liu and F. F. Liu, Advanced NDT\&E Techniques for composites, Aviation Industry Press, New Delhi, India, 2017.

[2] M. Gagné and D. Therriault, "Lightning strike protection of composites," Progress in Aerospace Sciences, vol. 64, pp. 1-16, 2014.

[3] K. B. Katnam, L. F. M. Da Silva, and T. M. Young, "Bonded repair of composite aircraft structures: a review of scientific challenges and opportunities," Progress in Aerospace Sciences, vol. 61, pp. 26-42, 2013.

[4] S. Kashif Ur Rehman, Z. Ibrahim, S. A. Memon, and M. Jameel, "Nondestructive test methods for concrete bridges: a review," Construction and Building Materials, vol. 107, pp. 58-86, 2016.

[5] P. Cawley and R. D. Adams, "An automated coin-tap technique for the non-destructive testing of composite structures," Composites Evaluation, vol. 55, pp. 11-15, 1987.

[6] P. Cawley, "The impedance method of non-destructive inspection," NDT International, vol. 17, no. 2, pp. 59-65, 1984.

[7] J. S. Leng, S. Y. Du, D. F. Wang, and Q. L. Li, "Sensitivity of the coin-tap method of nondestructive testing for composite structures," Acta Materiae Compositae Sinica, vol. 12, no. 4, pp. 99-105, 1995.

[8] S. Abrate, "Modeling of impacts on composite structures," Composite Structures, vol. 51, no. 2, pp. 129-138, 2001.

[9] J. C. Feng and Z. F. Yu, "Analytical model for stiffness of the low velocity impact for composite laminate with delaminations," Acta Materiae Compositae Sinica, vol. 35, no. 10, pp. 2768-2776, 2018.

[10] Z. P. Li, "A method for computing the deflection of elastic circular plates under concentrated loads," Chinese Quarterly of Mechanics, vol. 18, no. 1, pp. 96-99, 1982.

[11] A. W. Wang, "Solution to asymmetric bending of circular plates under single load by using point-source function," Chinese Journal of Theoretical and Applied Mechanics, vol. 24, no. 3, pp. 125-131, 1992.
[12] Z. Z. Dong, W. H. Peng, S. C. Li, and J. Liu, "Bending problems of solid circular plates under non-symmetrical load," Journal of China University of Mining \& Technology, vol. 61, no. 2, pp. 297-302, 2009.

[13] P. Cawley and R. D. Adams, "The mechanics of the coin-tap method of non-destructive testing," Journal of Sound and Vibration, vol. 122, no. 2, pp. 299-316, 1988.

[14] A. A. Shabana, Theory of Vibration: An Introduction, Springer, New York, NY, USA, 2018.

[15] S. J. Kim, "Damage detection in composite laminates using coin-tap method," Journal of the Acoustical Society of America, vol. 123, no. 5, p. 3064, 2008.

[16] P. Cawley and C. Theodorakopoulos, "The membrane resonance method of non-destructive testing," Journal of Sound and Vibration, vol. 130, no. 2, pp. 299-311, 1989.

[17] R. I. Mackie and A. E. Vardy, "Applying the coin-tap test to adhesives in civil engineering: a numerical study," International Journal of Adhesion and Adhesives, vol. 10, no. 3, pp. 215-220, 1990.

[18] T. Oh, J. S. Popovics, and S.-H. Sim, "Analysis of vibration for regions above rectangular delamination defects in solids," Journal of Sound and Vibration, vol. 332, no. 7, pp. 1766-1776, 2013.

[19] I. Solodov, J. Bai, and G. Busse, "Resonant ultrasound spectroscopy of defects: case study of flat-bottomed holes," Journal of Applied Physics, vol. 113, no. 22, pp. 26-31, 2013.

[20] S. Esola, I. Bartoli, S. E Horner, J. Q. Zheng, and A. Kontsos, "Defect detection via instrumented impact in thick-sectioned laminate composites," Journal of Nondestructive Evaluation, vol. 36, 2017.

[21] O. O. Ochoa and J. N. Reddy, Finite Element Analysis of Composite Laminates, Springer, Dordrecht, The Netherlands, 1992.

[22] Z. Y. Shi and X. H. Ding, Aircraft Structural Mechanics, National Defense Industry Press, Arlington, VA, USA, 2013. 